\title{
The Construction of A Sheep Acute Respiratory Distress Syndrome Model Induced by Smoke Inhalation Injury
}

\author{
Yu Fei ${ }^{1^{*}}$,Tan Yuan-fei ${ }^{1 *}$, Sheng Guan-nan ${ }^{2}$, Chen Guan-nan², Wang Nan-nan², Gong Ting ${ }^{2}$, Qu Bo ${ }^{3 \#}$ \\ 1.Emergency Department, the Seventh Affiliated Hospital, Sun Yat-sen University, Shen Zhen,518107;Yu Fei, Email \\ E-mail: doctor_yuf@126.com, https://orcid.org/0000-0001-5312-3328; Tan Yuan-fei, Email tyf.0604@163.com, \\ https://orcid.org/0000-0001-5985-6952 2.Jinzhou Medical University, Jin Zhou, 121002 \\ 2.Jinzhou Medical University, Jin Zhou,121002 \\ 3.Institute of Disaster Medicine, Tianjin University, Tian Jin, 300162 \\ *Yu Fei and Tan Yuan-fei are co-first authors. \#Qu Bo is the corresponding author. \\ Received:1 December 2019 Accepted: 5 December 2019 Published: 31 December 2019 \\ Cite this Article Yu Fei, Tan Yuan-fei, Sheng Guan-nan, Chen Guan-nan, Wang Nan-nan, Gong Ting, Qu Bo. \\ The construction of a sheep acute respiratory distress syndrome model induced by smoke inhalation injury \\ [J].Medical Research,2019.1(1): 1-11, Http://dx.doi.org/10.6913/MRHK.201912_1(1).0001 \\ Copyright (C) 2020 Creative Publishing Co., Limited. All rights reserved. Email: kycbshk@gmail.com.
}

\begin{abstract}
Objective To establish a model of acute respiratory distress syndrome induced by sheep smoke inhalation lung injury.

Methods Nine healthy, 12-24 month old, non-pregnant female sheep were randomly selected, the sheep were divided into three groups, through the internal control of smoke generator device components ( $\mathrm{CO}, \mathrm{CO} 2,02$, particle concentration and smoke temperature, etc.) to adjust the exposure time of the sheep model construction of smoke. The chest CT were examined before and after exposure to smoke at $6 \mathrm{~h}, 12 \mathrm{~h}, 24 \mathrm{~h}$ and $48 \mathrm{~h}$, respectively. From the smoke exposure $12 \mathrm{~h}$ endotracheal intubation and mechanical ventilation, by adjusting the ventilator parameters of hypoxemia and hypercapnia, statistical analysis of PaO2/FiO2, PEEP and other indicators, according to the ARDS definition of diagnosis in Berlin definition.
\end{abstract}

Results The CT showed mild inflammation of the lungs in first group, oxygen index of 95\% confidence interval [331.13, 353.53]; In second groups, oxygenation index 95\% confidence interval [220.09, 383.90]; CT showed airway edema, pulmonary inflammatory exudation and lung injury in third group. Oxygenation index 95\% confidence interval of [195.16, 244.84], PEEP $>5 \mathrm{~cm}$. There was significant difference between the two groups $(P<0.05)$.

Conclusions The severity of lung injury can be changed by adjusting the exposure time of smoke inhalation; when the smoke exposure time up to 45 minutes, a stable model of smoke inhalation lung injury in sheep ARDS can be successfully established.

Key words : Sheep; smoke inhalation injury; acute respiratory distress syndrome; model; mechanical ventilation

\section{INTRODUCTION}

The generation of human civilization by fire brings many conveniences to life, but at the same time it also brings people the hidden danger of fire. According to statistics, in the past ten years, there have been 180,000 fires and 1,698 deaths per year ${ }^{[1]}$.According to the American Burn Database, more than 200,000 burn patients were admitted from 2005 to $2014^{[2]} 30 \%$ of burn patients are associated with smoke inhalation lung injury, 
Medical Research ISSN 2664-0333 Volume 1 No.1 PP.1-11 http://dx.doi.org/10.6913/MRHK.201912_1(1).0001

Yu Fei, Tan Yuan-fei et al. The construction of a sheep acute respiratory distress syndrome model induced by smoke inhalation injury and the mortality rate of such patients increased by 24 times compared with patients with simple burns ${ }^{[3,4]} \cdot$.In addition, $20 \%$ of these patients will develop acute respiratory distress syndrome ${ }^{[3,5,6]}$. An average of 190,000 people are diagnosed with ARDS in the United States each year, with a mortality rate of $26-36 \%$, while domestically $>40 \%{ }^{[7]}$. These data indicate that SILI and the ARDS caused by it are imminent medical problems, which urgently need to be studied and solved by scholars.

At present, there is no authoritative international standard for the diagnosis, severity classification and prognosis of SILI, and the treatment methods are also varied. This also shows that people do not pay much attention to smoke injuries, and some related research is also basic research. There is an urgent need for a SILI rapid diagnosis method and an authoritative severity classification standard.

In 2012, the European Society of Critical Care Medicine proposed the ARDS "Berlin Definition"[8]. It provides a basis for the classification of ARDS, but there is no matching animal experimental model. The domestic SILI model has the following characteristics: mostly small animal models, no research reports on the SILI model of large animals; no uniform model establishment methods, such as the establishment of a cotton smoke inhalation acute lung injury rat model, the smoke inhalation made by the smoke generator Rat models of lung injury, etc .; most of them stay on the research of injury mechanism; there is no clear diagnostic criteria or classification criteria to determine the degree of lung injury. The foreign models are mainly large animals, and the most widely used one is still the goat SILI model that Hubbard GB has established in $1988^{[9]}$. Although this method has stabilized after continuous improvement, there are still some problems. Although the temperature of smoke is controlled within a certain range, it is seriously inconsistent with the actual situation of burns; in addition, the concentration of smoke cannot be controlled.

In order to better study the ARDS caused by SILI and provide theoretical and experimental support for its treatment, we designed and made an ARDS model of sheep smoke inhalation lung injury.

\section{MATERIALS AND METHODS}

Twelve 12-24-month-old female non-pregnant small-tailed Han sheep were selected, requiring a weight of $(30 \pm 5) \mathrm{kg}$, in good health, without fever, cough, diarrhea and other uncomfortable symptoms.The experiment was approved by the hospital ethics committee. All laboratory animals strictly abide by the "Guidebook for the Use and Care of Laboratory Animals" published by the National Institutes of Health in 1985. The feeding of laboratory animals is carried out in accordance with the Swiss Laboratory Animal Health Act.

Sheep selected in good condition 3 days before the experiment were placed in cages and fed individually, and $200 \mathrm{~g}$ of feed was given at 10:00 and 16:00 respectively, and water was continuously supplied. Fasting 36 hours before the experiment, drinking fast for the first 12 hours.

Medication before anesthesia, sedation (1 hour before anesthesia, intramuscular injection of midazolam $-0.05 \mathrm{mg} / \mathrm{kg}$ ), anticholinergic drugs (30 minutes before anesthesia: intramuscular injection of atropine $-0.05 \mathrm{mg} / \mathrm{kg}$ ); induced anesthesia, xylazine hydrochloride ( $\mathrm{Lu}$ Mianxin $0.1 \sim 0.15 \mathrm{ml} / \mathrm{kg}$ intramuscular injection), the anesthesia effect can be maintained for $2 \sim 3 \mathrm{~h}$. If there is a pain reaction, the drug can be added intravenously, and the additional amount is generally $1 / 5$ to $1 / 4$ of the first injection.

Turn on the air conditioner 2 hours in advance for preheating, to maintain the CT indoor temperature at 26 30 ${ }^{\circ} \mathrm{C}$, turn on the CT 15 minutes in advance, and prepare for the air meter. After the anesthesia was placed, the sheep was lifted to the CT room for CT examination. The head was placed in the prone position. The CT scan was performed before the smoke exposure to observe the CT performance of the lungs of normal sheep. At the same time, the existence of lung lesions was excluded.

Set the temperature parameter to $37^{\circ} \mathrm{C}$, and turn on the temperature control system (to make the temperature 
Medical Research ISSN 2664-0333 Volume 1 No.1 PP.1-11 http://dx.doi.org/10.6913/MRHK.201912_1(1).0001

Yu Fei, Tan Yuan-fei et al. The construction of a sheep acute respiratory distress syndrome model induced by smoke inhalation injury in the smoke box fluctuate less than $2{ }^{\circ} \mathrm{C}$ around the set parameter) to avoid the compound injury caused by smoke inhalation and thermal burn. The oxygen concentration fluctuates between 19-21\% according to the set value, which can avoid animal suffocation due to space constriction and lack of oxygen. Through the observation window of the smoke box, you can observe the reaction and breathing frequency of the tested animal. After the set time, remove the animal and fully expose it to the air.

After the sheep get out of the smoke, they are transferred to the intensive care unit of the animal, and then they are monitored (the ECG monitor continuously monitors the pulse oxygen saturation and heart rate, the tee connects to the animal physiological monitor to continuously monitor the arterial blood pressure), and the intravenous fluid $(10 \%$ glucose $1000 \mathrm{ml}+0.9 \%$ Sodium chloride injection $500 \mathrm{ml}+$ sodium potassium magnesium calcium glucose injection $500 \mathrm{ml}+10 \%$ potassium chloride $10 \mathrm{ml}$, prepare low molecular weight dextran injection and lactated Ringer's solution), $0.5-1 \mathrm{ml}$ Lu Mianning intravenously every two hours. Lower urinary tube, stomach tube.Mechanical ventilation: adopt volume control mode, initial setting (tidal volume $\mathrm{TV}=10 \mathrm{ml} / \mathrm{kg}$, respiratory rate $\mathrm{RR}=12$ times $/ \mathrm{min}$, maintain $\mathrm{PCO} 2$ at $35-45 \mathrm{mmHg}$ by adjusting respiratory rate, $\mathrm{PEEP}=5 \mathrm{~cm}, \mathrm{FiO} 2=21 \%$ ) The goal is to maintain the airway platform pressure Pplat $<35 \mathrm{mmH} 2 \mathrm{O}$; the goal to correct hypercapnia is: $\mathrm{PaCO} 2<60, \mathrm{pH}>7.2$, increase the respiratory rate by 2 times / min, up to 30 times / min, if Pplat $>35 \mathrm{mmH} 2 \mathrm{O}$, Then reduce TV1ml $/ \mathrm{kg}$ to maintain Pplat $=$ $35 \mathrm{mmH} 2 \mathrm{O}$; the goal of correcting hypoxemia is: $\mathrm{PaO} 2>70, \mathrm{SpO} 2>92 \%$, if the arterial blood gas analysis suggests $\mathrm{SpO} 2<92 \%$, then adjust by increasing $\mathrm{FiO} 2$ by $10 \%$ Until $\mathrm{SpO} 2>92 \%$, refer to the ARDSNet PEEP titration table; the goal of correcting hypocapnia: $\mathrm{PaCO} 2>30, \mathrm{pH}<7.5$, reduce the respiratory rate by 2 times / $\mathrm{min}$, the slowest is 10 times / $\mathrm{min}$, at $1 \mathrm{ml} /$ The rate of $\mathrm{kg}$ reduces the tidal volume, and the final tidal volume cannot be lower than $4 \mathrm{ml} / \mathrm{kg}$.

Intraoperative infusion used compound sodium lactate Ringer injection, sodium chloride injection, hydroxyethyl starch (130/0.4) electrolyte injection, $10 \%$ glucose injection, sodium potassium magnesium calcium glucose injection. The input liquid is mainly crystal liquid, the total amount of hydroxyethyl starch is not more than $10 \mathrm{ml} / \mathrm{kg}$, and the total amount of daily rehydration is not less than $75 \mathrm{ml} / \mathrm{kg}$. Intraoperative blood pressure was maintained at more than $80 \%$ of basal blood pressure, CVP range was $10-15 \mathrm{~mm} \mathrm{Hg}$ and or PAWP range was $15-20 \mathrm{~mm} \mathrm{Hg}$. During the experiment, actively correct the acid-base balance and electrolyte disorders, adjust the depth of anesthesia and control blood pressure.

\section{STATISTICAL METHOD}

Statistical analysis was performed using SPSS17.0 statistical software, and quantitative data was expressed as mean \pm standard deviation $\overline{\mathrm{X}} \pm \mathrm{s}$; comparison between groups was analyzed by variance; $\mathrm{P}<0.05$ indicated that the difference was statistically significant.

\section{RESULT}

Nine sheep were purchased from the Experimental Animal Center of the Chinese Academy of Military Medical Sciences and divided into three groups (Group A, Group B, and Group C), each group containing 3 sheep. Table 1 is the basic situation of experimental sheep.

Table 1 Basic situation of experimental animals

\begin{tabular}{c|c}
\hline index & data \\
\hline Age $($ month $)$ & $17.20 \pm 4.18$ \\
\hline Gender $(\mathrm{M} / \mathrm{F})$ & $0 / 6$ \\
\hline Body height $(\mathrm{cm})$ & $80.53 \pm 3.49$ \\
\hline Body length $(\mathrm{cm})$ & $82.01 \pm 5.98$ \\
\hline Weight $(\mathrm{kg})$ & $32.4 \pm 4.7$ \\
\hline Heart rate $(\mathrm{bpm})$ & $90 \pm 5$ \\
\hline
\end{tabular}


Medical Research ISSN 2664-0333 Volume 1 No.1 PP.1-11 http://dx.doi.org/10.6913/MRHK.201912_1(1).0001

Yu Fei, Tan Yuan-fei et al. The construction of a sheep acute respiratory distress syndrome model induced by smoke inhalation injury

\begin{tabular}{c|c}
\hline Breathing frequency $($ times / min $)$ & $15 \pm 4$ \\
\hline Body temperature $\left({ }^{\circ} \mathrm{C}\right)$ & $38.7 \pm 1.5$ \\
\hline
\end{tabular}

Note: All data in Table 1 except $x$ is the corresponding quantity is expressed as $\overline{x \pm s}$

The first group of sheep set a smoke exposure time of 15 minutes, and the main monitoring index results are as follows: all three sheep in this group continuously monitored 48h heart rate (HR), blood pressure (systolic pressure, diastolic pressure, average arterial pressure), and respiratory rate (BR ), Blood oxygen saturation (SpO2) is relatively stable, blood oxygen saturation is always higher than 90, blood gas analysis results are shown in Table 2, caused by acute lung injury can not meet the ARDS diagnostic criteria, that is, oxygenation index $>300$.

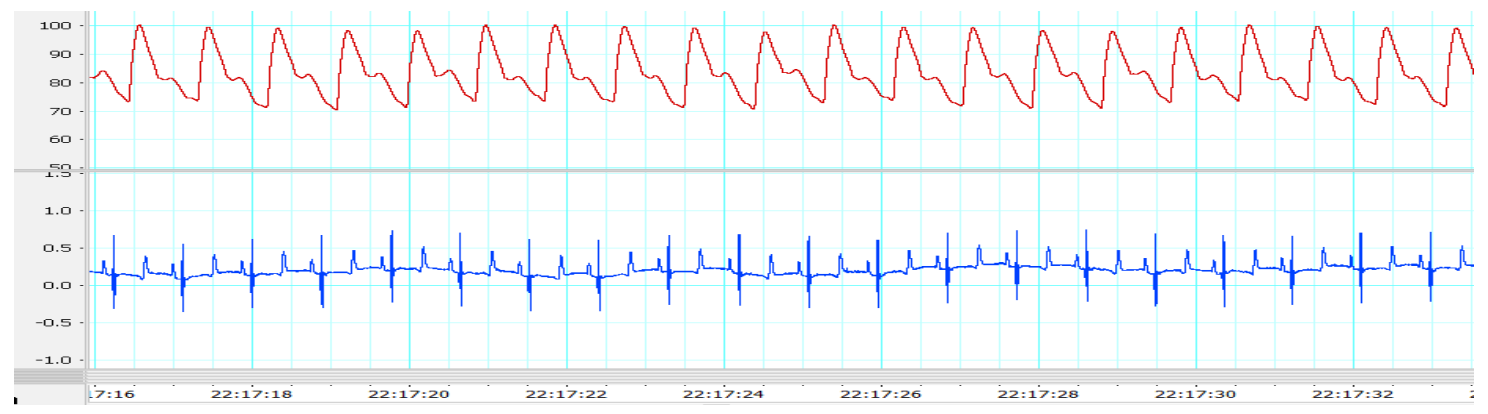

Figure 1 Results of heart rate and blood pressure monitoring (excerpt)

Figure 2 CT examination results

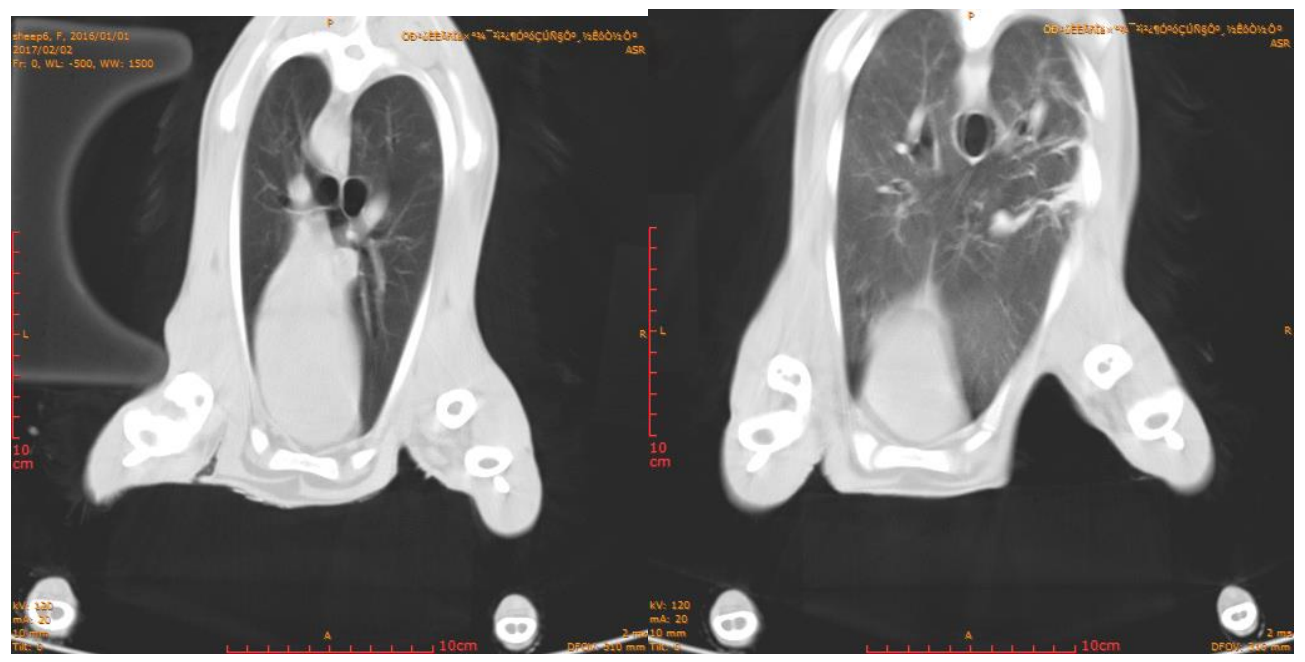

A Before smoke exposure

B 6h after smoke exposure

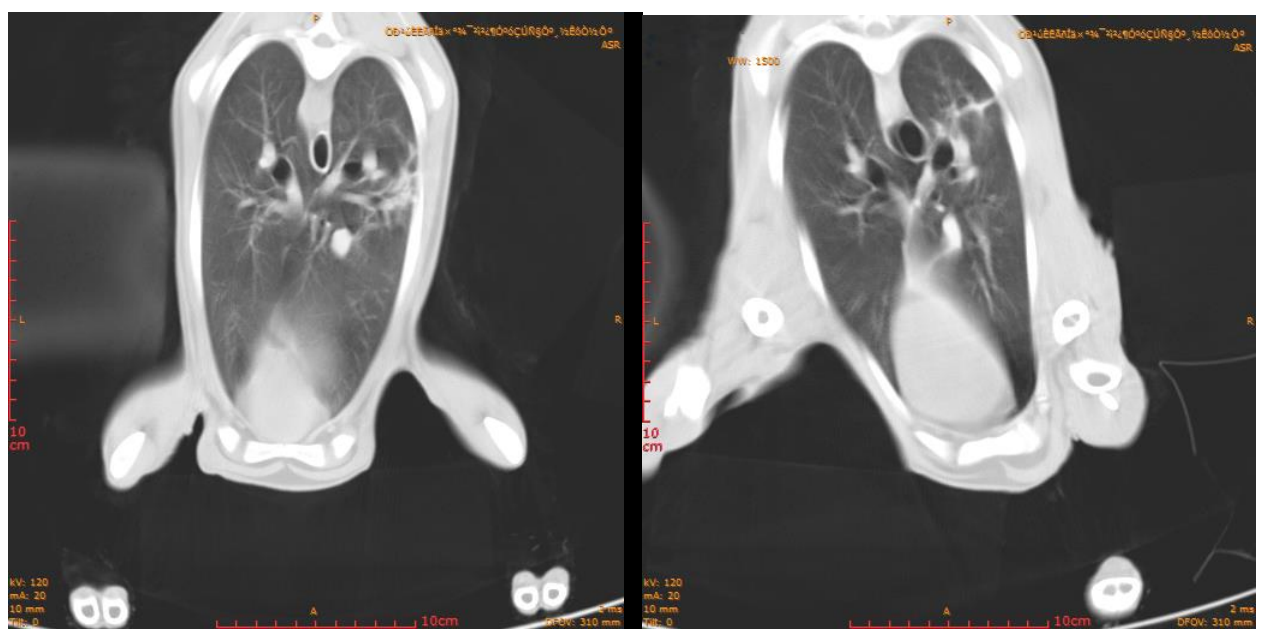

C 12h after smoke exposure

D 24h after smoke exposure 
Medical Research ISSN 2664-0333 Volume 1 No.1 PP.1-11 http://dx.doi.org/10.6913/MRHK.201912_1(1).0001 Yu Fei, Tan Yuan-fei et al. The construction of a sheep acute respiratory distress syndrome model induced by smoke inhalation injury Table 2 Blood gas analysis and oxygenation index results

\begin{tabular}{cccccc}
\hline & $12 \mathrm{~h}$ & $18 \mathrm{~h}$ & $24 \mathrm{~h}$ & $30 \mathrm{~h}$ & $36 \mathrm{~h}$ \\
\hline $\mathrm{pH}$ & $7.476 \pm 0.011$ & $7.43 \pm 0.015$ & $7.417 \pm 0.008$ & $7.391 \pm 0.034$ & $7.385 \pm 0.056$ \\
$\mathrm{PCO} 2$ & $35 \pm 3$ & $37 \pm 2$ & $41 \pm 1$ & $40 \pm 3$ & $40 \pm 2$ \\
$\mathrm{SO} 2$ & $94 \pm 1$ & $95 \pm 1$ & $96 \pm 1$ & $95 \pm 1$ & $94 \pm 1$ \\
$\mathrm{FiO} 2$ & 21 & 21 & 21 & 21 & 21 \\
$\mathrm{PO} 2$ & $70 \pm 1$ & $72 \pm 1$ & $74 \pm 1$ & $76 \pm 1$ & $79 \pm 1$ \\
$\mathrm{PO} 2 / \mathrm{FiO} 2$ & $337 \pm 6$ & $342 \pm 5$ & $352 \pm 3$ & $363 \pm 4$ & $376 \pm 6$ \\
\hline
\end{tabular}

The smoke exposure time of the second group of sheep was set at 30 minutes. The main monitoring indicators were as follows: all three sheep in this group continuously monitored $48 \mathrm{~h}$ heart rate (HR), blood pressure (systolic pressure, diastolic pressure, average arterial pressure), and respiratory rate (BR), blood oxygen saturation ( $\mathrm{SpO} 2)$. One of the sheep showed symptoms of shortness of breath and wheezing. The blood gas analysis results are shown in Table 3. The sheep met the ARDS diagnostic criteria, that is, the oxygenation index was $<300$. The remaining two did not experience discomfort and did not meet the ARDS diagnostic criteria.

Figure 3 Heart rate and blood pressure monitoring results (excerpt)

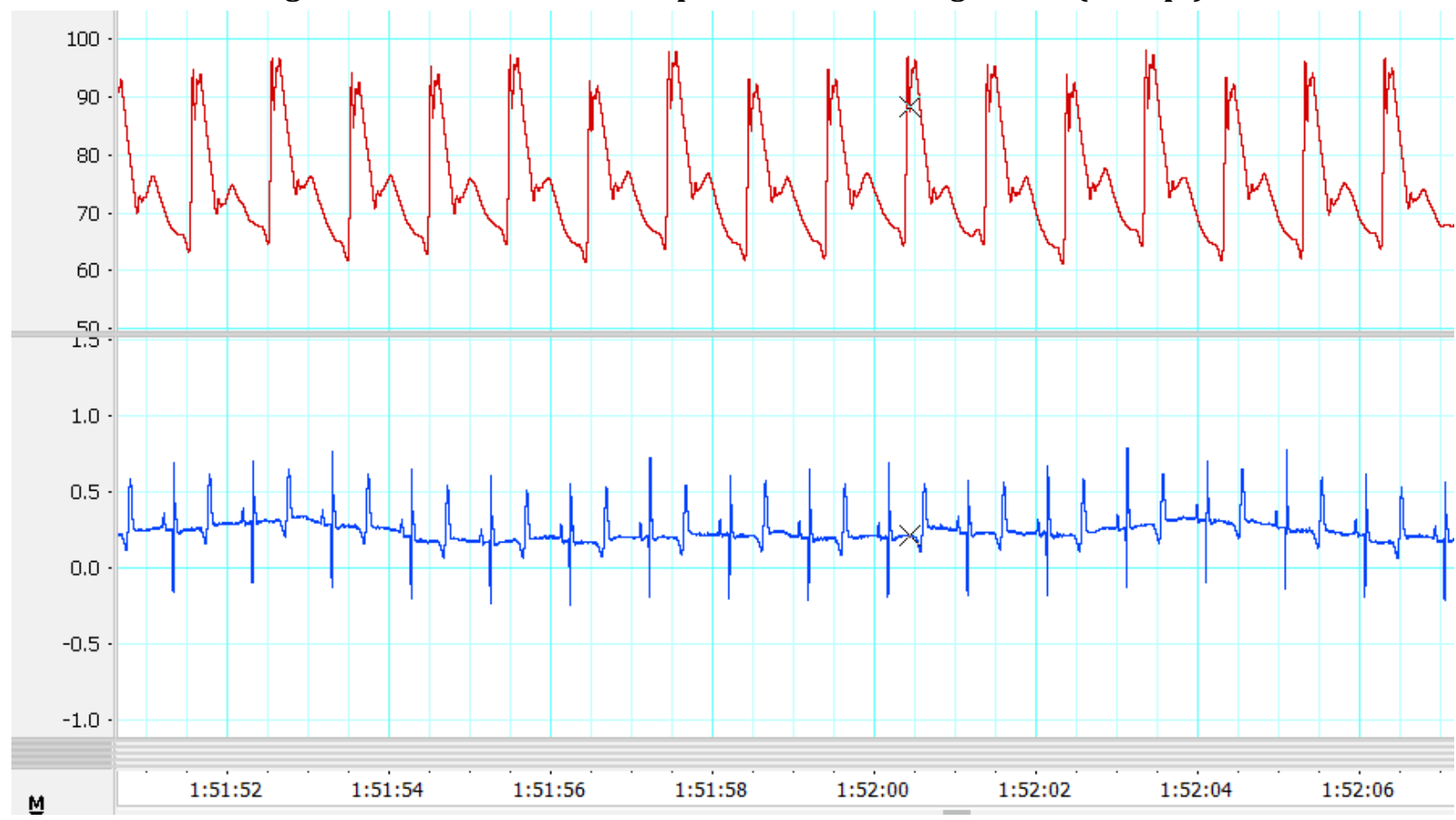


Medical Research ISSN 2664-0333 Volume 1 No.1 PP.1-11 http://dx.doi.org/10.6913/MRHK.201912_1(1).0001

Yu Fei, Tan Yuan-fei et al. The construction of a sheep acute respiratory distress syndrome model induced by smoke inhalation injury

Figure 4 CT examination results

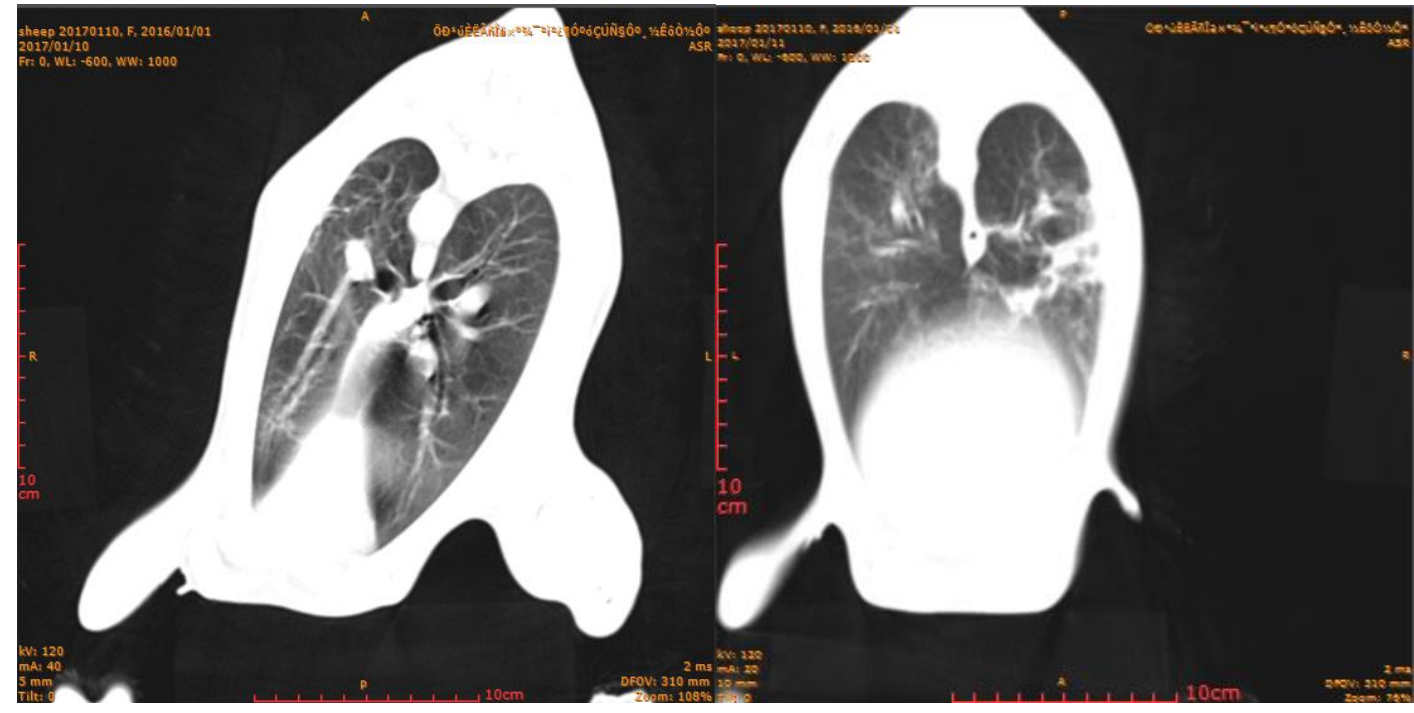

A Before smoke exposure

B 6h after smoke exposure

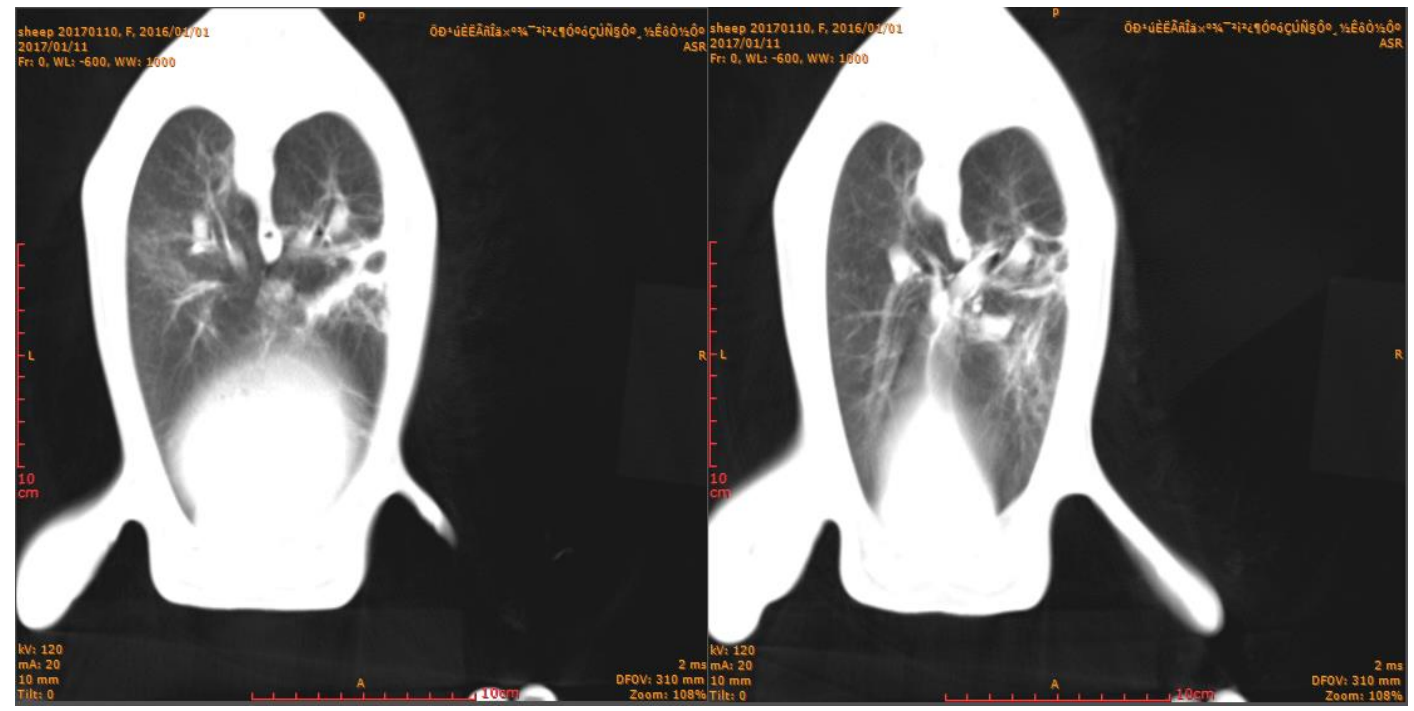

C 12h after smoke exposure

D 24h after smoke exposure

Table 3 Blood gas analysis and oxygenation index results

\begin{tabular}{cccccc}
\hline & $12 \mathrm{~h}$ & $18 \mathrm{~h}$ & $24 \mathrm{~h}$ & $30 \mathrm{~h}$ & $36 \mathrm{~h}$ \\
\hline $\mathrm{pH}$ & $7.480 \pm 0.037$ & $7.416 \pm 0.011$ & $7.380 \pm 0.026$ & $7.389 \pm 0.019$ & $7.415 \pm 0.064$ \\
$\mathrm{PCO} 2$ & $34 \pm 3$ & $37 \pm 3$ & $43 \pm 2$ & $40 \pm 2$ & $41 \pm 5$ \\
$\mathrm{SO} 2$ & $92 \pm 1$ & $93 \pm 1$ & $92 \pm 1$ & $91 \pm 1$ & $90 \pm 1$ \\
$\mathrm{FiO} 2$ & 21 & 21 & 21 & 21 & 21 \\
$\mathrm{PO} 2$ & $62 \pm 2$ & $65 \pm 2$ & $64 \pm 2$ & $65 \pm 2$ & $66 \pm 2$ \\
$\mathrm{PO} 2 / \mathrm{FiO} 2$ & $298 \pm 9$ & $307 \pm 10$ & $303 \pm 12$ & $310 \pm 11$ & $315 \pm 8$ \\
\hline
\end{tabular}

The smoke exposure time set for the third group of sheep is 45 minutes. The main monitoring index results are as follows: 
Medical Research ISSN 2664-0333 Volume 1 No.1 PP.1-11 http://dx.doi.org/10.6913/MRHK.201912_1(1).0001

Yu Fei, Tan Yuan-fei et al. The construction of a sheep acute respiratory distress syndrome model induced by smoke inhalation injury

Figure 5 Heart rate and blood pressure monitoring results (excerpt)

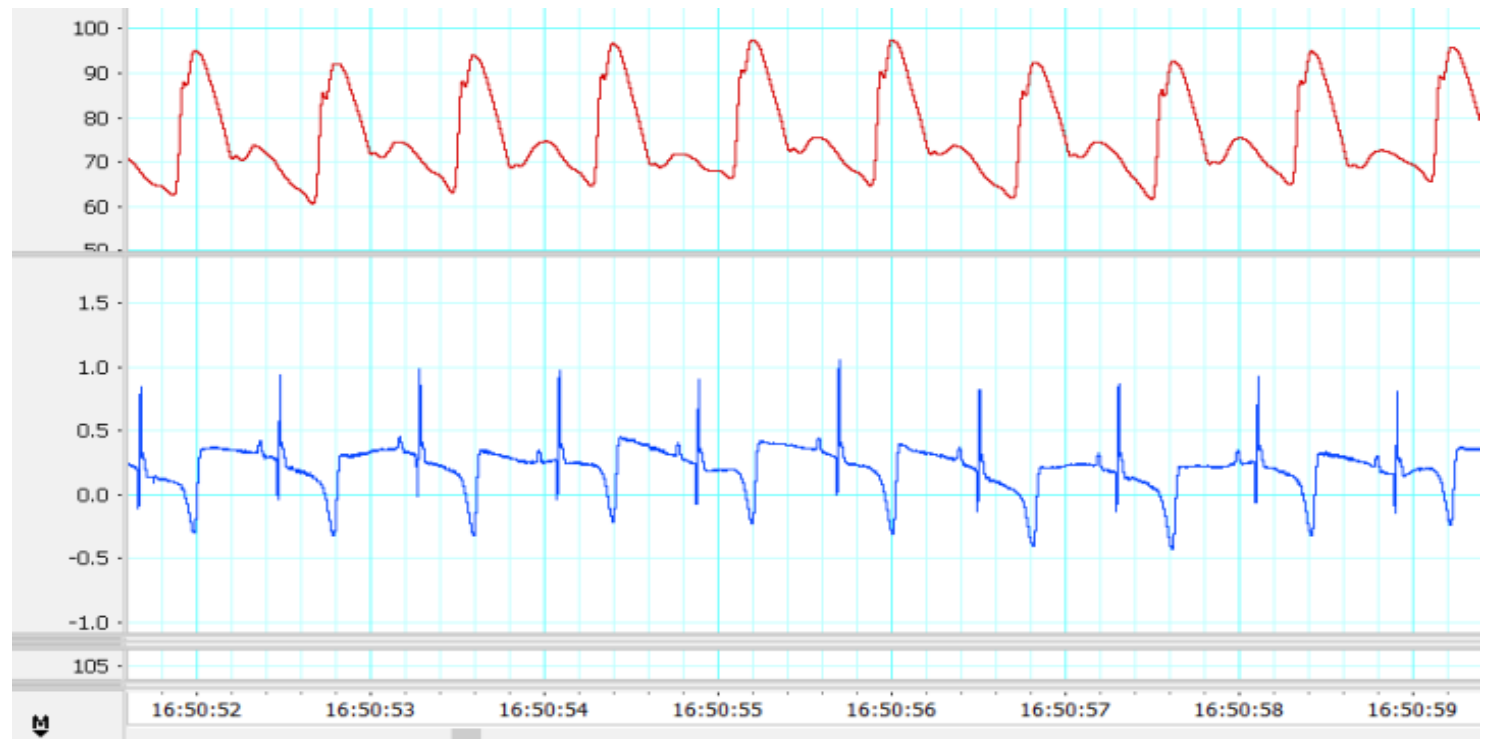

Figure 6 CT examination results

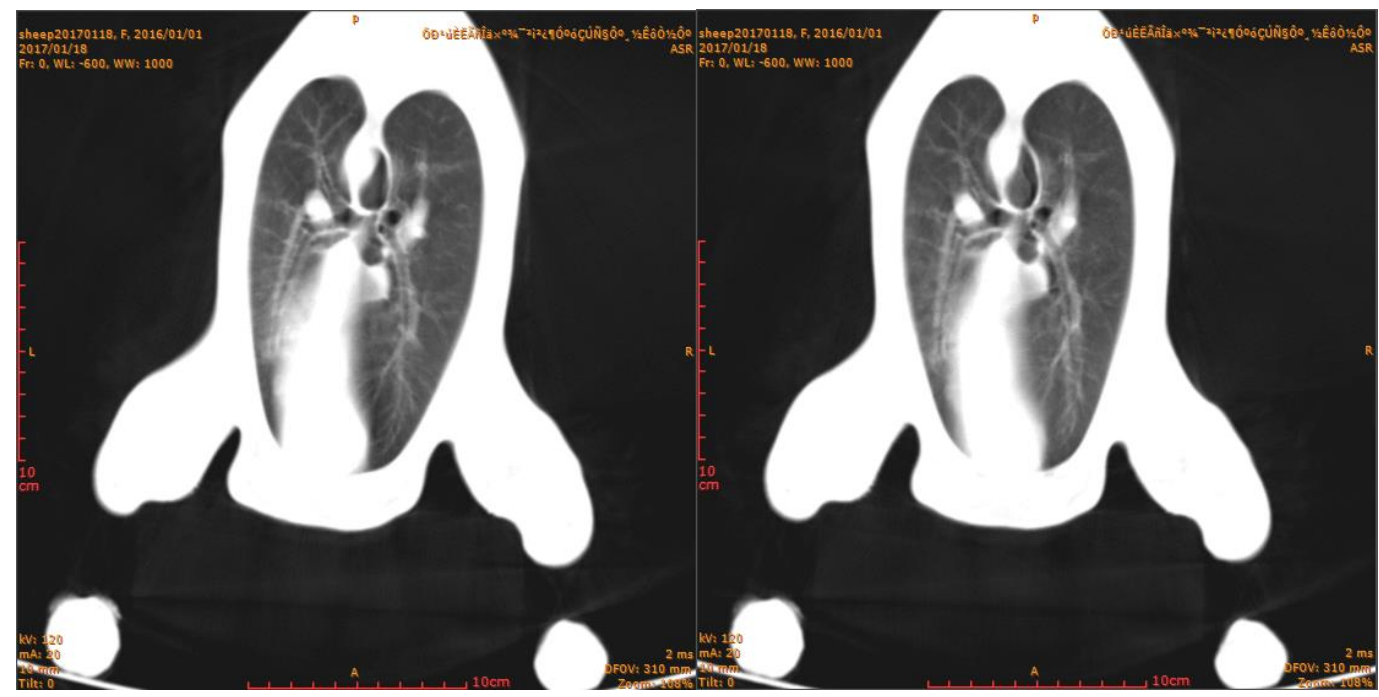

A Before smoke exposure

B 6h after smoke exposure

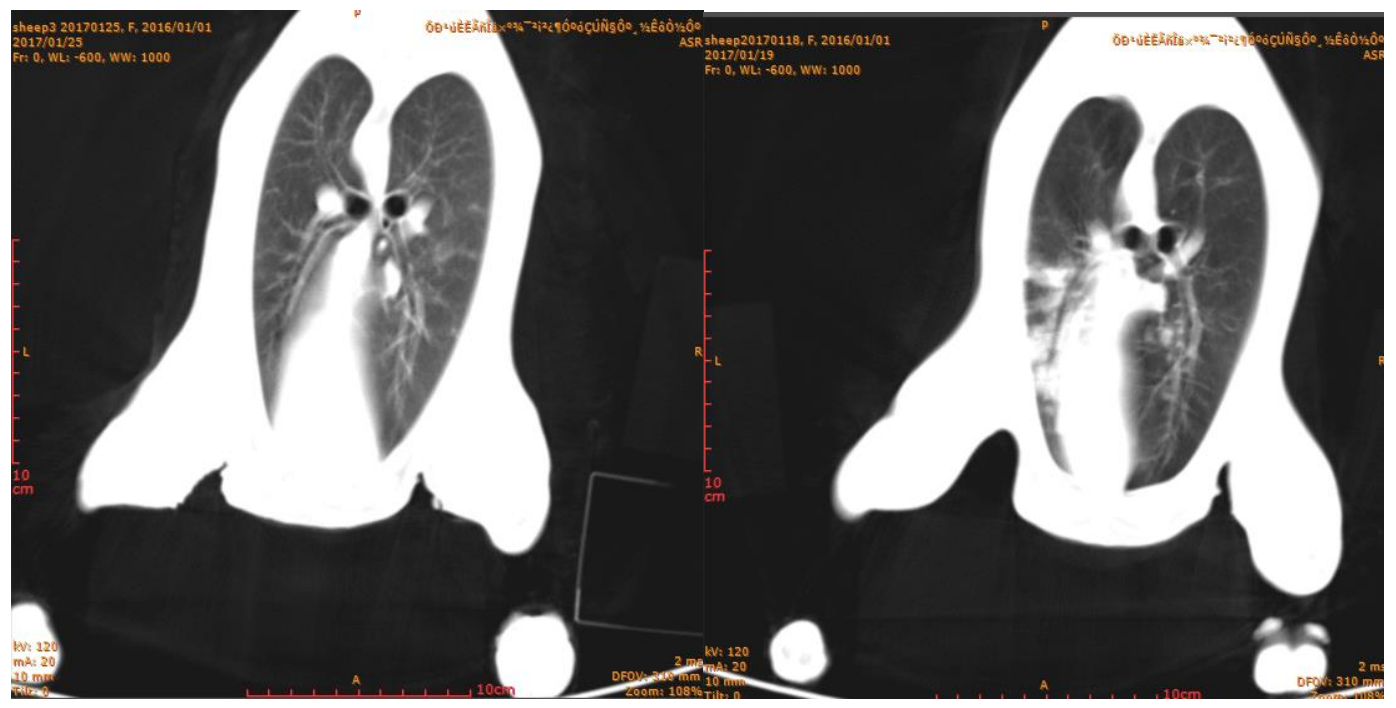

C 12h after smoke exposure

D 24h after smoke exposure 
Medical Research ISSN 2664-0333 Volume 1 No.1 PP.1-11 http://dx.doi.org/10.6913/MRHK.201912_1(1).0001

Yu Fei, Tan Yuan-fei et al. The construction of a sheep acute respiratory distress syndrome model induced by smoke inhalation injury

Figure 7 Visual observation of lung tissue and pathological results
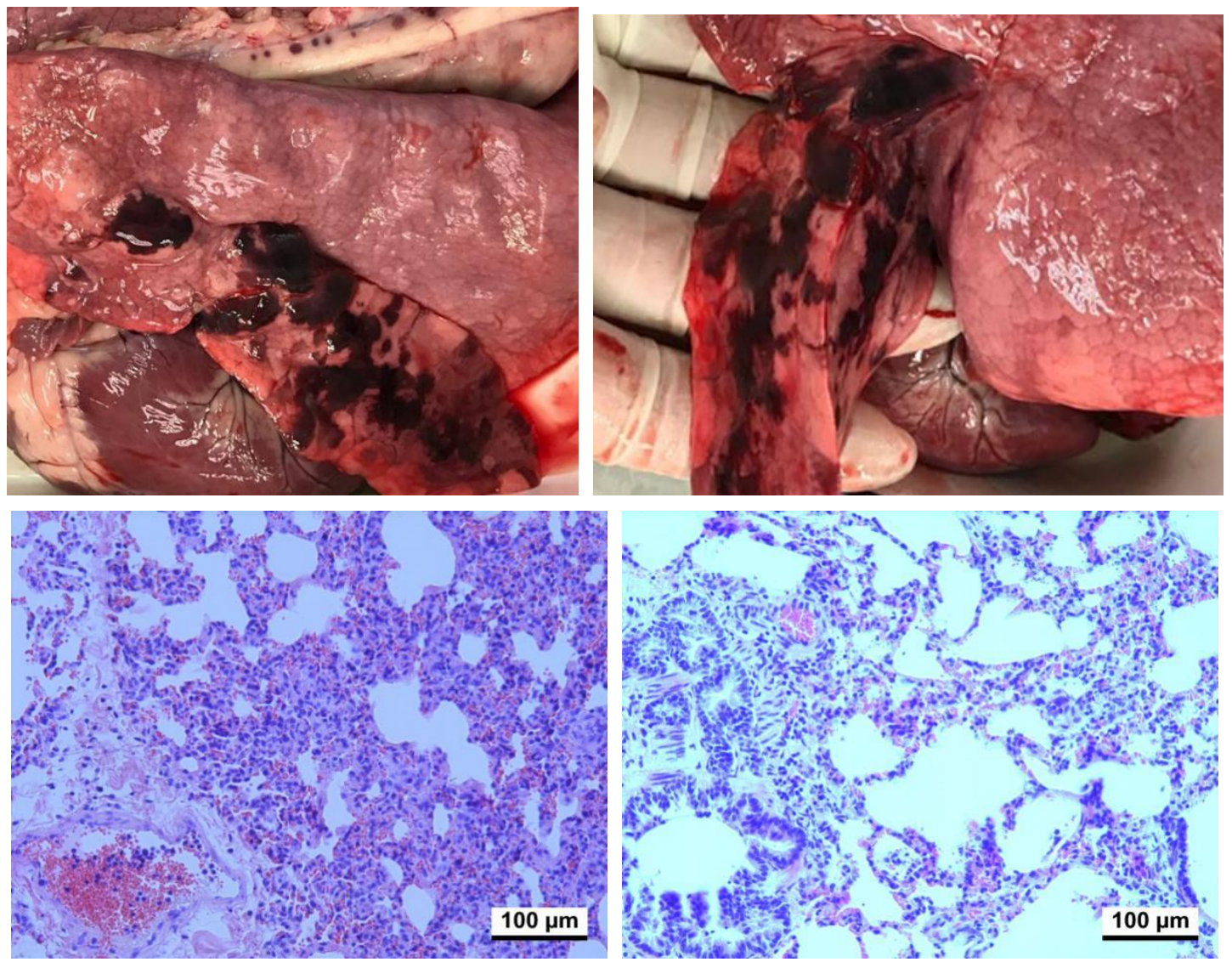

Table 4 Blood gas analysis and oxygenation index results

\begin{tabular}{cccccc}
\hline & $12 \mathrm{~h}$ & $18 \mathrm{~h}$ & $24 \mathrm{~h}$ & $30 \mathrm{~h}$ & $36 \mathrm{~h}$ \\
\hline $\mathrm{pH}$ & $7.428 \pm 0.014$ & $7.294 \pm 0.240$ & $7.357 \pm 0.011$ & $7.426 \pm 0.029$ & $7.433 \pm 0.333$ \\
$\mathrm{PCO} 2$ & $32 \pm 2$ & $36 \pm 1$ & $44 \pm 2$ & $42 \pm 3$ & $45 \pm 3$ \\
$\mathrm{SO} 2$ & $92 \pm 1$ & $93 \pm 1$ & $92 \pm 1$ & $91 \pm 1$ & $90 \pm 1$ \\
$\mathrm{FiO} 2$ & 21 & 21 & 21 & 21 & 21 \\
$\mathrm{PO} 2$ & $62 \pm 2$ & $65 \pm 2$ & $64 \pm 2$ & $65 \pm 2$ & $66 \pm 2$ \\
$\mathrm{PO} 2 / \mathrm{FiO} 2$ & $248 \pm 8$ & $220 \pm 10$ & $200 \pm 10$ & $211 \pm 4$ & $223 \pm 8$ \\
\hline
\end{tabular}

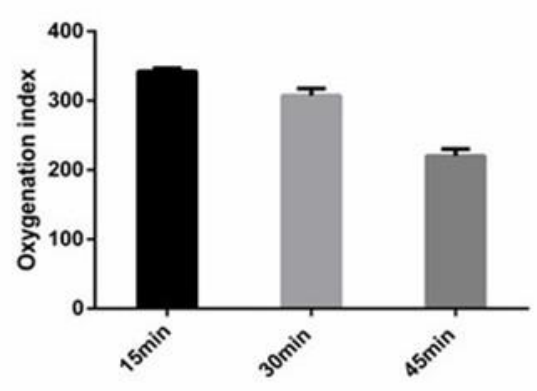

A 12h after exposure to smoke

\section{Oxygenation index}

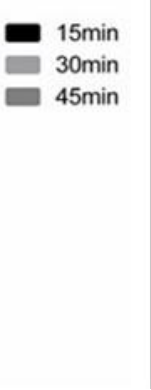

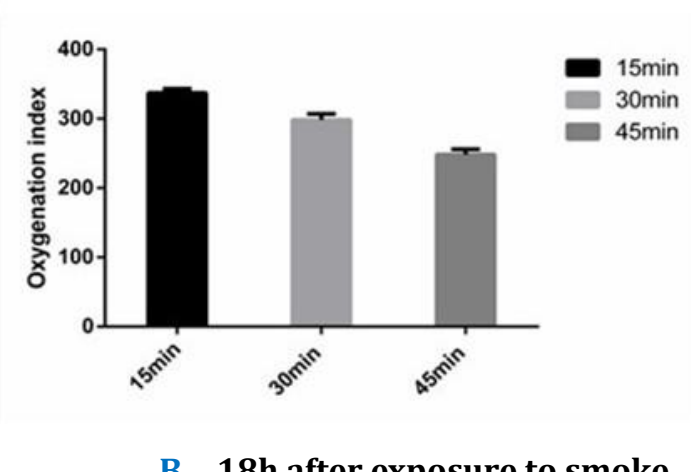

B 18h after exposure to smoke 


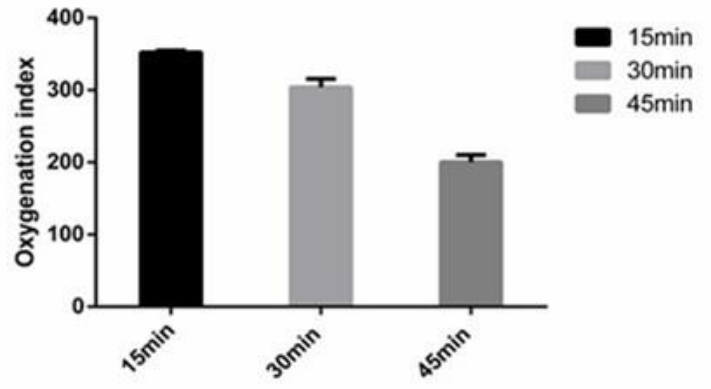

C $24 \mathrm{~h}$ after exposure to smoke

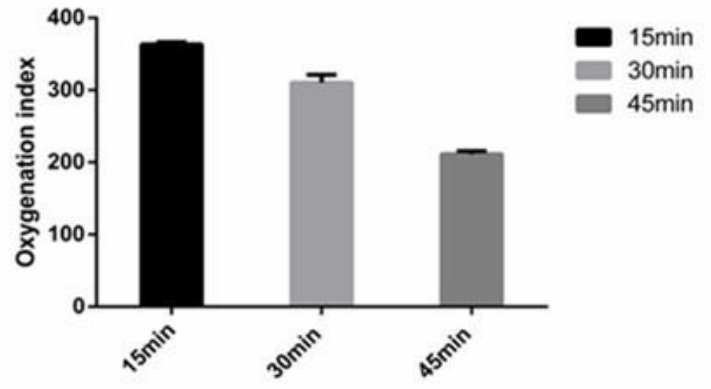

D $30 \mathrm{~h}$ after exposure to smoke

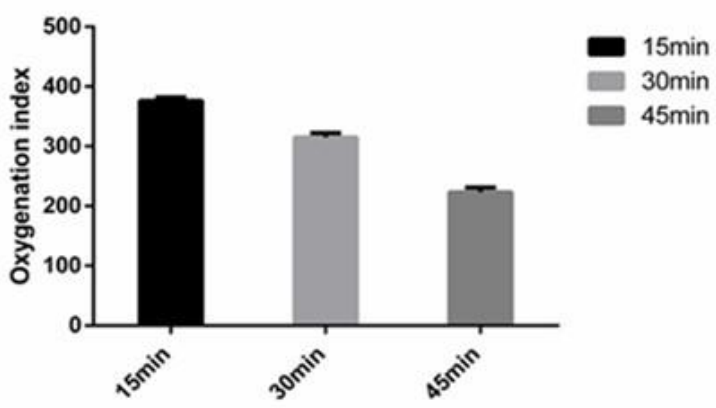

E 36h after exposure to smoke

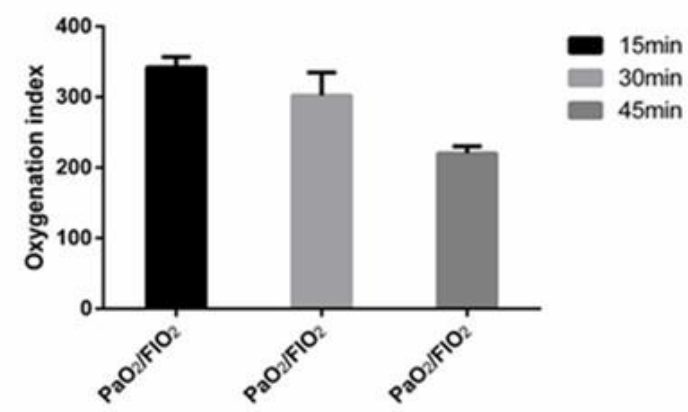

F $\quad 48 \mathrm{~h}$ after exposure to smoke

Note: The comparison of the average number of oxygenation indexes among multiple groups is by analysis of variance. The oxygenation index of each group after mechanical ventilation $(\mathrm{P}<0.05)$

According to the Berlin definition standard, the diagnosis results of the three groups of sheep are as follows: The 95\% confidence interval of the oxygenation index of the smoke exposure group for 15 minutes: [331.13, 353.53]; The 95\% confidence interval of the oxygenation index of the smoke exposure group for 30 minutes: [220.09, 383.90]; The 95\% confidence interval for the oxygen exposure index of the smoke exposure group for 45 minutes: [195.16, 244.84].Among them, the 95\% confidence interval of the oxygenation index in the 45min group is within the ARDS "Berlin definition" diagnostic criteria, and the 95\% confidence interval of the oxygenation index in the 30min group is within the ARDS "Berlin definition" diagnostic criteria, and the oxygenation index is $95 \%$ in the 15 min group The confidence interval is outside the ARDS "Berlin Definition" diagnostic criteria.

\section{DISCUSSION}

Burns can not only cause pathophysiological changes of a certain system or a single organ, but also cause the destruction of the function and structure of multiple organ systems ${ }^{[10]}$. Because burn is a complex injury that can cause damage to multiple organs, its complex pathophysiological process cannot be captured in in vitro experiments.Smoke inhalation lung injury In accidental burns, the incidence rate is $20 \%-30 \%$, and some of these patients will develop ARDS with a mortality rate of up to $40 \%{ }^{[3,7,11]}$.In order to study the impact of burns on various aspects, scholars have established a large number of animal burn models in the past few decades $^{[12]}$.The main function and purpose of these models is to study the pathophysiological mechanism and treatment of burns.It is particularly important to Understand the advantages and limitations of these models for the treatment of human-related diseases.

The experimental animals used in the smoke inhalation lung injury model are: mice, rats, rabbits, sheep, dogs, pigs, etc ${ }^{[12]}$. Rats dominate in China, abroad is dominated by sheep ${ }^{[13-16]}$. Rats have the characteristics of fast 
Medical Research ISSN 2664-0333 Volume 1 No.1 PP.1-11 http://dx.doi.org/10.6913/MRHK.201912_1(1).0001

Yu Fei, Tan Yuan-fei et al. The construction of a sheep acute respiratory distress syndrome model induced by smoke inhalation injury reproduction speed, low economic cost, easy modeling, easy operation, good repeatability and stability, etc $^{[12]}$.In particular, many studies have shown that certain organ structures, physiological characteristics, and immune mechanisms of rats are similar to humans. The tolerance of rats to hypoxia is higher than that of mice. Blood samples can be collected continuously for a certain period of time after injury to make dynamic observations. The pathological specimens are larger and can meet the requirements for the number of specimens in the experiment. It is widely used in research. However, the body shape of rats and humans is quite different. Many new scientific and technological achievements equipment (such as ECMO, Da Vinci robots, etc.) have not been miniaturized and cannot be applied to rats, which limits the application of new technologies and new technologies in experiments. Clinically instructive experimental research. Large animals are closer to humans first in shape and structure, and their tolerance is similar to humans in the process of injury. When large animals are used in the modeling of burn injury or smoke inhalation lung injury, they are closer to human ALI / ARDS after smoke inhalation lung injury in physiology, pathogenesis, pathology, and pathophysiology ${ }^{[17]}$.Abdullahi $\mathrm{A}$, in reviewing smoke inhalation lung injury in burn animal models, proposed that sheep are the gold standard for smoke inhalation lung injury ${ }^{[12]}$. The goat smoke inhalation lung injury model established in 1988, which has been continuously used in foreign experimental research, although this model can control the temperature of the smoke within a certain range $\left(35^{\circ} \mathrm{C}-40{ }^{\circ} \mathrm{C}\right)$, it is seriously inconsistent with the actual situation of the burn site. In addition, although the model can determine the composition of each component in smoke (such as $\mathrm{CO}, \mathrm{CO} 2, \mathrm{O} 2$, particles, etc.), it cannot control the concentration of each component in smoke within a certain range. Finally, the various animal models currently available are mainly used to study acute lung injury (ALI) caused by smoke inhalation, which is a relatively mild stage of lung injury, and may even involve ARDS, but there is no model that specifically studies ARDS caused by smoke inhalation ${ }^{[18]}$.The high mortality rate of ARDS caused by smoke inhalation injury and its impact on the prognosis of burns are all problems that people need to solve urgently.

This study uses the second-generation smoke generation device self-developed by the previous research group ${ }^{[19,20]}$, real-time control and adjustment of indoor temperature (in the smoke generator cabin), temperature in the box, humidity in the box, operating time, smoke concentration, etc. When the smoke exposure time was 15 minutes, a chest CT scan revealed lung inflammation, bronchial edema, and exudation. However, the vital signs and other monitoring indicators of the sheep did not change significantly, nor did the respiratory dysfunction appear. And after 12 hours from smoke exposure, the oxygenation index is always greater than 300, and its 95\% confidence interval is [331.13, 353.53], which does not meet the ARDS diagnostic criteria. It shows that the smoke exposure time of 15 minutes can cause lung damage, but the severity of the damage is less. When the smoke exposure time is 30 minutes, one of the sheep can be diagnosed with ARDS. When the smoke exposure time is 45 minutes, all can be diagnosed as ARDS, and the $95 \%$ confidence interval of its oxygenation index is [195.16, 244.84]. It shows that when the smoke exposure time is 45 minutes, a stable ARDS model of smoke inhalation lung injury can be formed. Analysis of variance of the oxygenation index of the three groups of sheep showed that $\mathrm{P}<0.05$, that is, the differences between the groups were statistically significant, indicating that as the smoke exposure time was prolonged, the degree of sheep lung damage gradually increased.

This experiment successfully obtained a stable model of ARDS caused by smoke inhalation lung injury, and further confirmed that lung injury during burns is mainly due to particles and toxic components in the inhaled gas, and the degree of lung injury and the time of smoke exposure Proportional to. The ARDS model directly mimics the clinical pathophysiology of human smoke injury, making it flexible for clinical treatment of burns. The model currently does not have an accurate severity classification for ARDS caused by smoke inhalation lung injury, but with the deepening of research, its classification of smoke inhalation lung injury severity and exploration of ARDS treatment will be of great significance. 
Medical Research ISSN 2664-0333 Volume 1 No.1 PP.1-11 http://dx.doi.org/10.6913/MRHK.201912_1(1).0001

Yu Fei, Tan Yuan-fei et al. The construction of a sheep acute respiratory distress syndrome model induced by smoke inhalation injury

\section{ACKNOWLEDGEMENT}

This study was supported by Tianjin Science and Technology Plan Project (No:15ZXLCSY00040).

The research was performed in Institute of Disaster Medicine and Public Health, Affiliated Hospital of

Logistics University of People's Armed Police Force.

\section{STATEMENT}

There is no conflict of interest in this article.

\section{REFERENCES}

1 FU Zhi-min. Analysis on the fire data in China for the period between 2003-2012 [J]. Journal of Safety and Environment, 2014, 6): $341-5$.

2 BAQUI Z, LI W, ZAKHARY E, et al. Inferior Vena Cava Filter Utilization for Burn Patients in the United States: Analysis of the National Burn Repository [J]. Journal of Vascular Surgery, 2015, 61(6): 103S-4S.

3 MCCLURE J, MOORE E C. Burns and inhalational injury [J]. Anaesthesia \& Intensive Care Medicine, 2017, 12(9): 393-8.

4 Liu Wei, Jin Faguang, Pan Lei, et al. Retrospective analysis of the treatment of 52 cases smoke inhalation acute lung injury [J]. Chinese Journal of Lung Diseases(Electronic Edition), 2014, 7(3): 79-80.

5 KREYER S, SCARAVILLI V, LINDEN K, et al. Early Utilization of Extracorporeal CO2 Removal for Treatment of Acute Respiratory Distress Syndrome Due to Smoke Inhalation and Burns in Sheep [J]. Shock, 2016, 45(1): 65.

6 REPER P, HEIJMANS W. High-frequency percussive ventilation and initial biomarker levels of lung injury in patients with minor burns after smoke inhalation injury [J]. Burns, 2015, 41(1): 65-70.

7 ENKHBAATAR P, JR P B, SUMAN O, et al. Pathophysiology, research challenges, and clinical management of smoke inhalation injury [J]. Lancet, 2016, 388(10052): 1437.

8 RANIERI V M, RUBENFELD G D, THOMPSON B T, et al. Acute respiratory distress syndrome: the Berlin Definition [J]. JAMA, 2012, 307(23): 2526-33.

9 HUBBARD G B, SHIMAZU T, YUKIOKA T, et al. Smoke inhalation injury in sheep [J]. American Journal of Pathology, 1988, 133(3): 660 .

10 Zhou Jinxiu, Jiang Zhengying, Liu Mingzhu, et al. Advances in the research of diagnosis and treatment of ventilator induced induced lung injury after burn[J]. Chinese Journal of Burns, 2020, 36(02):137-141.

11 DRIES D J, ENDORF F W. Inhalation injury: epidemiology, pathology, treatment strategies [J]. Scandinavian Journal of Trauma, Resuscitation and Emergency Medicine, 2013, 21(1): 31.

12 ABDULLAHI A, AMINI-NIK S, JESCHKE M G. Animal models in burn research [J]. Cellular \& Molecular Life Sciences Cmls, 2014, 71(17): 3241.

13 HAN Zhihai, DUAN Yunyou, JIANG Yi, et al. The establishment of cotton smoke inhalation-induced acute lung injury model in rats [J]. Translational Medicine Journal, 2014, 5): 274-7.

14 HE Kaiming, DAI Tianyang, ZEN Peiyuan. Experimental study of the protective effect of perfluorocarbon on lung ischemia reperfusion injury in rats[J]. Journal of Southwest Medical University, 2019,42(1):31-35.

15 LIU Yi-fan, TANG Hong-wei, WU Xiao-li, et al. Reproduction and evaluation of a rat model of inhalation lung injury caused by black gunpowder smog [J]. Medical Journal of Chinese People's Liberation Army, 2013, 38(8): 670-4.

16 Zhou Min, Yao Bin, Yan Shangxue, et al. Establishment of a rat model of inhalation lung injury induced by powder combustion [J]. Medical Journal of Chinese People's Liberation Army, 2005, 30(8): 734-7.

17 BALLARD-CROFT C, WANG D, SUMPTER L R, et al. Large-animal models of acute respiratory distress syndrome [J]. Annals of Thoracic Surgery, 2012, 93(4): 1331.

18 LIU N T, SALTER M G, KHAN M N, et al. Closed-Loop Control of FiO2 Rapidly Identifies Need For Rescue Ventilation and Reduces ARDS Severity in a Conscious Sheep Model of Burn and Smoke Inhalation Injury [J]. Shock, 2017, 47(2): 200.

19 CAO J, HOU S, HUI D, et al. In VivoTracking of Systemically Administered Allogeneic Bone Marrow Mesenchymal Stem Cells in Normal Rats through Bioluminescence Imaging [J]. Stem Cells International, 2016, 2016(3): 1-11.

20 SONG M, LV Q, ZHANG X, et al. Dynamic Tracking Human Mesenchymal Stem Cells Tropism following Smoke Inhalation Injury in NOD/SCID Mice [J]. Stem Cells International, 2016, 2016(1): 1-13. 